\title{
A NOTE ON GROUPS WITH NON-CENTRAL NORM
}

\author{
by R. A. BRYCE and L. J. RYLANDS
}

(Received 22 June, 1992)

1. Introduction. The norm $\kappa(G)$ of a group $G$ is the subgroup of elements of $G$ which normalize every subgroup of $G$. Under the name kern this subgroup was introduced by Baer [1]. The norm is Dedekindian in the sense that all its subgroups are normal. A theorem of Dedekind [5] describes the structure of such groups completely: if not abelian they are the direct product of a quaternion group of order eight and an abelian group with no element of order four. Baer [2] proves that a 2-group with non-abelian norm is equal to its norm.

As for the embedding of the norm Schenkman [10] proves that $\kappa(G)$ is always contained in the second centre $\zeta_{2}(G)$ of $G$. A recent result of one of us and Cossey and Ormerod [3], extending a result of Meixner [9], concerns the restriction that a non-central norm imposes on a group: a metabelian group $G$ of exponent $p^{2}$ where $p$ is prime, and in which $\kappa(G)$ is not central, has nilpotency class at most $2 p-2$. (In some sense this is the natural direction of generalization of Baer's result (op. cit.) for, when $p$ is odd, the norm is necessarily abelian.) However neither exponent $p^{2}$ nor metabelian can be simply omitted from this result if the desired result is bounded class. Indeed consider the group $G=A \times B$ where $A$ has exponent $p$ and $B$ has exponent $p^{2}$ with $\kappa(B)$ not central; for example, let $B$ be non-abelian of exponent $p^{2}$ and order $p^{3}$. Then it is easy to see that $\kappa(G)=\zeta_{1}(A) \times \kappa(B) \neq \zeta_{1}(G)$. However for suitably large primes $p$ the group $A$, and therefore the group $G$, need not even be nilpotent, and certainly there is no bound on its class if it is nilpotent. Hence metabelian cannot simply be omitted. Choosing $A=$ $C_{p} \operatorname{wr}\left(C_{p} \times C_{p} \times \ldots\right)$ and $B=\left\langle a, b: a^{p^{3}}=b^{p^{2}}=1, b^{-1} a b=a^{p+1}\right\rangle$ gives a group $G$ which is metabelian, of exponent $p^{3}$, with $\kappa(G)$ not central, but which is not nilpotent.

The present note observes that the first counter-example above fails when $p$ is three, because then $A$ is of class at most three. This is a result of Levi [8]; see M. Hall [6, p. 322] for a proof. We prove in fact that no counter-example can be made in this case.

For convenience we denote by $\mathscr{G}$ the class of groups $G$ of exponent dividing 9 in which $\kappa(G) \neq \zeta_{1}(G)$.

TheOREM 1.1. A group in $\mathscr{G}$ has nilpotency class at most 5, and this bound is best possible.

2. The class $\mathscr{H}$. It will be useful to recall the definition of the Hughes subgroup in the present context. If $G$ is a group then $H_{3}(G)$ is the subgroup of $G$ generated by elements whose order is not 3. Therefore the elements in $G \backslash H_{3}(G)$, if any, all have order exactly three. We will denote by $\mathscr{H}$ the class of groups of exponent 9 for which $H_{3}(G) \neq G$. Strauss and Szekeres [11] showed that the well-known question of Hughes [7] has a positive answer in the case of the prime 3 . We note this in our case as follows.

Lemma 2.1 (Strauss and Szekeres [11]). If $H \in \mathscr{H}$ then $\left|H: H_{3}(H)\right|=3$.

The next result is effectively in Strauss and Szekeres but for completeness we record a proof. We adopt as a continuing notation that in a group $H \in \mathscr{H}, H_{3}(H)$ is denoted by D.

This work was supported by an Australian Research Council Grant.

Glasgow Math. J. 36 (1994) 37-43. 
Lemma 2.2. Let $H \in \mathscr{H}$. Then for every $y \in D$, the normal closure of $y$ in $H$ is abelian.

Proof. Let $y \in D$ and $x \notin D$. Then $x y$ and $x^{2} y$ are neither in $D$. Consequently $x, x y$ and $x^{2} y$ all have order 3 . Therefore

$$
1=(x y)^{3}=x y x y x y=x^{-2} y x^{2} \cdot x^{-1} y x \cdot y=y^{x^{2}} y^{x} y
$$

and, similarly,

$$
1=y^{x} y^{x^{2}} y
$$

It follows that

$$
y^{x} y^{x^{2}}=y^{x^{2}} y^{x}
$$

and hence

$$
\left[y^{x}, y\right]=1 .
$$

Now for $d \in D, x d^{-1} \notin D$ so, from (2.4),

$$
1=\left[y^{x d^{-1}}, y\right]^{d}=\left[y^{x}, y^{d}\right] \text {. }
$$

In this we may replace $x$ by $x^{2}$, and get $\left[y^{x^{2}}, y^{d}\right]=1$. But then from (2.3) we deduce that $\left[y^{d}, y\right]=1$. Combined with (2.4) this means that for all $h$ in $H,\left[y^{h}, y\right]=1$. Finally for all $h_{1}, h_{2} \in H$,

$$
\left[y^{h_{1}}, y^{h_{2}}\right]=\left[y^{h_{1} h_{2}^{-1}}, y\right]^{h_{2}}=1
$$

as required.

Lemma 2.5. For $H \in \mathscr{H}, D$ has class at most 3 and $\gamma_{3}(D)$ has exponent dividing 3.

Proof. By (2.2) for all $y_{1}, y_{2} \in D$,

$$
\begin{aligned}
1 & =\left[y_{1}^{y_{2}}, y_{1}\right]=\left[y_{1}^{-1} y_{1}^{y_{2}}, y_{1}\right] \\
& =\left[y_{1}, y_{2}, y_{1}\right] .
\end{aligned}
$$

It follows from a result of Levi [8] (see p. 322 of M. Hall [6] for a proof) that $D$ has class 3 at most and that $\gamma_{3}(D)$ has exponent dividing 3 .

Lemma 2.6. Let $H \in \mathscr{H}$. Suppose that $y \in \gamma_{i}(D)$ for some $i \geq 0$. If $x \in H \backslash D$ then for some $a \in \gamma_{i}(D)$,

$$
[y, x, x]=a^{3} .
$$

Proof. For, by (2.3), and using (2.2)

$$
\begin{aligned}
1 & =y^{2}\left(y^{x^{2}} y^{x} y\right) y^{-2}=y^{2} y^{x^{2}} y^{x} y^{-1}=y^{2} y^{x^{2}} y^{-1} y^{x} \\
& =y^{3}\left[y, x^{2}\right][y, x] \\
& =y^{3}[y, x]^{3}[y, x, x] \\
& =(y[y, x])^{3}[y, x, x]
\end{aligned}
$$

which yields the required result.

Lemma 2.7. For $H \in \mathscr{H}, y \in D$ and $h \in H$

$$
\left[h, y^{-1}\right]=[h, y]^{-1} \text {. }
$$


Proof. Note that

$$
\left[h, y^{-1}\right]=h^{-1} y h y^{-1}=y^{-1} h^{-1} y h
$$

by $(2.2)$, whence

$$
\left[h, y^{-1}\right]=[y, h]=[h, y]^{-1} .
$$

Lemma 2.8. For $H \in \mathscr{H}, x \in H \backslash D, y_{1} \in \gamma_{i}(D), y_{2} \in \gamma_{j}(D)$ and $y_{3} \in D$, we have

(i) $\left[\left[y_{1}, x\right],\left[y_{2}, x\right]\right]=a^{3}$, for some $a \in \gamma_{i+j}(D)$,

and

(ii) $\left[y_{1}, x, y_{2}, x\right]=b^{3}$, for some $b \in \gamma_{i+j}(D)$,

(iii) $\left[y_{1}, x, y_{2}, y_{3}, x\right]=1$.

Proof. From (2.2) we have $1=\left[y_{1} y_{2}, x, y_{1} y_{2}\right]$. Using standard commutator identities we conclude, on expanding, that

$$
1=\left[y_{1}, x, y_{2}\right]\left[y_{2}, x, y_{1}\right]
$$

since all other terms are trivial by (2.2). Replacing $y_{1}$ by $\left[y_{1}, x\right]$ gives

$$
1=\left[y_{1}, x, x, y_{2}\right]\left[\left[y_{2}, x\right],\left[y_{1}, x\right]\right] \text {. }
$$

Therefore

$$
\begin{aligned}
{\left[\left[y_{1}, x\right],\left[y_{2}, x\right]\right] } & =\left[y_{1}, x, x, y_{2}\right] \\
& =\left[a_{0}^{3}, y_{2}\right] \\
& =\left[a_{0}, y_{2}\right]^{3}
\end{aligned}
$$

for some $a_{0}$ in $\gamma_{i}(D)$, by (2.2) and (2.6). This verifies (i).

(ii) From the Jacobi-type Hall identity we get

$$
\begin{aligned}
{\left[y_{1}, x, y_{2}, x\right] } & =\left[y_{1}, x, y_{2}, x\right]^{y_{2}^{-1}} \\
& =\left[x,\left[y_{1}, x\right]^{-1}, y_{2}^{-1}\right]^{-\left[y_{1}, x\right]}\left[y_{2}^{-1}, x^{-1},\left[y_{1}, x\right]\right]^{-x} \\
& =\left[x,\left[y_{1}, x\right]^{-1}, y_{2}\right]\left[y_{2}, x^{-1},\left[y_{1}, x\right]\right]^{x}
\end{aligned}
$$

by (2.2), (2.6) and (2.7). Therefore

$$
\begin{aligned}
{\left[y_{1}, x, y_{2}, x\right] } & =\left[y_{1}, x, x, y_{2}\right]\left[\left[y_{2}, x\right]^{-1},\left[y_{1}^{x}, x\right]\right] \\
& =\left[a_{0}^{3}, y_{2}\right] b_{0}^{3} \\
& =\left(\left[a_{0}, y_{2}\right] b_{0}\right)^{3},
\end{aligned}
$$

for some $a_{0} \in \gamma_{i}(D)$ and some $b_{0} \in \gamma_{i+j}(D)$, by (2.2), (2.6) and (i). This is what (ii) requires.

(iii) Now (2.9) holds for all $x$ in $H$, not just for $x \notin D$ and it may be written in the form $1=\left[x, y_{1}, y_{2}\right]\left[x, y_{2}, y_{1}\right\}$. In particular therefore we have

$$
\begin{aligned}
{\left[y_{1}, x, y_{2}, y_{3}\right] } & =\left[y_{2},\left[y_{1}, x\right], y_{3}\right]^{-1} \\
& =\left[y_{2}, y_{3},\left[y_{1}, x\right]\right] \\
& =\left[\left[y_{1}, x\right],\left[y_{2}, y_{3}\right]\right]^{-1},
\end{aligned}
$$


whence

$$
\begin{aligned}
{\left[y_{1}, x, y_{2}, y_{3}, x\right] } & =\left[y_{1}, x,\left[y_{2}, y_{3}\right], x\right]^{-1} \\
& \in \gamma_{3}(D)^{3} \\
& =1
\end{aligned}
$$

by (ii) and (2.5).

This completes the proof of (2.8).

There is a close connexion between the classes $\mathscr{G}$ and $\mathscr{H}$. One aspect of this is given in (3.2) below. Another is alluded to in the second example given in Section 1. We formulate this here in the context of this note.

Lemma 2.10. Let $H \in \mathscr{H}$ and let $K$ be non-abelian of order 27 and exponent 9. Then there is a group $G$ in $G$ and a sub-direct embedding $G \rightarrow H \times K$.

Proof. Choose $x_{0} \in H \backslash D$ and let $K=\left\langle a, b: a^{9}=b^{3}=1, b^{-1} a b=a^{4}\right\rangle$. Define $G$ to be the subgroup $D\left\langle a^{3}, b\right\rangle\left\langle x_{0} a\right\rangle$ of $H \times K$. It is left as an exercise for the reader to verify that $b \in \kappa(G) \backslash \zeta_{1}(G)$.

Once Theorem 1.1 is proved, $(2.10)$ will allow us to conclude that groups in $\mathscr{H}$ have class at most 5 . In fact we will prove the following result.

THEOREM 2.11. Every group in $\mathscr{H}$ has class at most 5 and this bound is best possible.

3. Non-central norm. Recall that $\mathscr{G}$ is the class of all groups $G$ of exponent dividing 9 in which $\kappa(G)$ is not central.

Lemma 3.1. Let $G \in \mathscr{G}$. Then if $w \in \kappa(G) \backslash \zeta_{1}(G)$ and $C=C_{G}(w)$, (i) $|G: C|=3$ or $G$ has class at most 4 , and (ii) $[G, w]=\left\langle x^{3}\right\rangle$, for every $x \in G \backslash C$ if $G$ is not of class 4 .

Proof. For every $x \in G \backslash C,[w, x]=x^{ \pm 3}$ since $w \in \kappa(G) \backslash \zeta_{1}(G)$. Hence every element of $G / \zeta_{1}(G)$ not in $C / \zeta_{1}(G)$ has order 3. Therefore $H_{3}\left(G / \zeta_{1}(G) \subseteq C / \zeta_{1}(G) \neq G / \zeta_{1}(G)\right.$. By Strauss and Szekeres [11] therefore, either $3=\left|G / \zeta_{1}(G): H_{3}\left(G / \zeta_{1}(G)\right)\right| \geqslant$ $\left|G / \zeta_{1}(G): C / \zeta_{1}(G)\right| \geqslant 3$ and it follows that $|G: C|=3$ as required by (i); or $G / \zeta_{1}(G)$ has exponent 3 and therefore $G$ has class at most 4 by Levi [8]. Part (ii) now follows immediately.

Corollary 3.2. For every $G \in \mathscr{G}$ of class greater than $4, G /[G, w] \in \mathscr{H}$ and $H_{3}(G /[G, w])=C /[G, w]$, for every $w \in \kappa(G) \backslash \zeta_{1}(G)$.

For fixed $w \in \kappa(G) \backslash \zeta_{1}(G)$ we denote $C_{G}(w)$ by $C$ and $[G, w]$ by $X$ throughout.

Lemma 3.3. Let $G \in \mathscr{G}$ have class greater than 4 and $y \in C$. Then for every $g \in G$,

$$
\left[g, y^{3}\right]=[g, y]^{3} \text {. }
$$

Proof. Since by (3.2), $G / X \in \mathscr{H}$, we have from (2.2) that $[g, y, y] \in X \subseteq \zeta_{1}(G)$. Hence, again using (2.2),

$$
\begin{aligned}
{\left[g, y^{3}\right] } & =[g, y]\left[g, y^{2}\right]^{y} \\
& =[g, y][g, y]^{y}[g, y]^{y^{2}} \\
& =[g, y]^{3}[g, y, y]\left[g, y, y^{2}\right] \\
& =[g, y]^{3}[g, y, y]^{3} \\
& =[g, y]^{3}
\end{aligned}
$$

as required. 
Lemma 3.4. For $G \in \mathscr{G}, x \notin C$ and $y_{1}, y_{2}, y_{3} \in C$ we have either $\gamma_{5}(G)=1$ or

$$
\left[y_{1}, y_{2}, x, y_{3}\right]^{3}=\left[y_{1}, x, y_{2}, y_{3}\right]^{3}=1 \text {. }
$$

Proof. Define $Z$ by $Z / X=\zeta_{1}(G / X)$. Note that $Z \subseteq \zeta_{2}(G)$. We prove first that if $G$ has class greater than 4 , then $Z$ is contained in $C$ and has exponent 3 .

Suppose that $G$ has class greater than 4 . Then $Z \subseteq C$ because, if not, there exists $x_{0} \in Z \backslash C$ and, by (3.1), $G=C\left\langle x_{0}\right\rangle$. However $C$ has class at most 4 by (3.2) and (2.5). Therefore every commutator of weight at least 5 whose entries are $x_{0}$ or belong to $C$ is trivial (recall that $x_{0} \in \zeta_{2}(G)$ ), contradicting the assumption that $G$ has class greater than 4. It follows that $Z \subseteq C$ as claimed.

Suppose that $z \in Z$ and choose $x \in G \backslash C$. Then $x z \notin C$ and so by (3.1) (ii) $(x z)^{3}=x^{ \pm 3}$. However

$$
(x z)^{3}=x^{3} z^{x^{2}} z^{x} z=x^{3} z^{3}\left[z, x^{2}\right][z, x]=x^{3} z^{3}[z, x]^{3}=x^{3} z^{3}
$$

Therefore $x^{3} z^{3}=(x z)^{3}=x^{ \pm 3}$, whence $z^{3}=1$ or $z^{3}=x^{3}$. Since $z^{2}$ also is in $Z$ and has order the same as $z$, we must have that $z^{3}=1$ or $z^{6}=\left(z^{2}\right)^{3}=x^{3}=z^{3}$, whence $z^{3}=1$. It follows that $Z$ has exponent 3 .

The result will follow when we show that $\left[y_{1}, y_{2}, x, y_{3}\right]$ and $\left[y_{1}, x, y_{2}, y_{3}\right]$ are in $Z$. Note first that, by (2.5), both $\left[y_{1}, y_{2}, x, y_{3}, y\right]$ and $\left[y_{1}, x, y_{2}, y_{3}, y\right]$ are in $X$ for every $y \in C$. Secondly consider $\left[y_{1}, y_{2}, x, y_{3}, x\right]$ and $\left[y_{1}, x, y_{2}, y_{3}, x\right]$. Both are in $X$ by (2.8)(ii) and (iii). Putting both these facts together we have the result claimed, that $\left[y_{1}, y_{2}, x, y_{3}\right]$ and $\left[y_{1}, x, y_{2}, y_{3}\right]$ are in $Z$. This completes the proof of (3.4).

We are now in a position to begin the proof-proper of Theorem 1.1. It suffices to show that every simple commutator of weight six whose entries are, in some order, $t_{1}, t_{2}$, $t_{3}, t_{4}, t_{5}, t_{6}$ is trivial. Since $C$ has class at most 4 by (3.2) and (2.5) we may as well assume that one of $t_{1}, \ldots, t_{6}$ is not in $C$. Renaming if necessary we may suppose it to be $t_{6}$ which we now call $x$. A different $t_{j}$ will have the property that precisely one of $t_{j}, t_{j} x$ and $t_{j} x^{-1}$ is in $C$. Whichever it is, call it $y_{j}(1 \leq j \leq 5)$. Since $\left\{t_{1}, t_{2}, \ldots, t_{6}\right\}$ and $\left\{y_{1}, y_{2}, \ldots, y_{5}, x\right\}$ generate the same group we need only show that every simple commutator of weight 6 with entries from the latter set is trivial. Without loss of generality we may suppose that its first entry is $y_{1}$.

LEMma 3.5. If exactly three of the unspecified entries in $\left[y_{1},-,-,-,-, x\right]$ or $\left[y_{1},-,-,-,-, y_{2}\right]$ are $x$ then each commutator is trivial.

Proof. Since the missing entries contain three $x$ 's, both entries of one pair of adjacent entries are $x$. Hence by (2.6), (3.2) and (3.3) the commutators in question will take one of the forms

$$
\left[y_{1}, x, y_{2}, x\right]^{3} \text { or }\left[y_{1}, y_{2}, x, x\right]^{3}
$$

on the one hand, and

$$
\left[y_{1}, x, y_{2}, y_{3}\right]^{3} \text { or }\left[y_{1}, y_{2}, x, y_{3}\right]^{3}
$$


on the other. The latter two are trivial by (3.4); and the first two are likewise trivial by (2.8)(ii) and (3.2), and (2.5) and (3.2) respectively.

Lemma 3.6. Both commutators $\left[y_{1}, x, x, x, x, x\right]$ and $\left[y_{1}, x, x, x, x, y\right]$ are equal to 1 .

Proof. This follows from (2.6) and (3.3).

It is now sufficient to show that the following result holds.

LEMMA 3.7. Every commutator of the form $\left[y_{1},-,-,-,-\right]$ in which at most two of the unspecified entries are equal to $x$ is central in $G$.

Proof. By (3.2) we can work in $H=G / X$, assuming the $y$ 's are in $D=C / X$ and $x \in H \backslash D$, and show that the commutators in question are trivial.

If at most one unspecified entry is $x$ then the commutator is trivial by $(2.5)$. So suppose exactly two are equal to $x$. By $(2.6),(2.8)$ the commutator is equal to $d^{3}$ for some $d \in \gamma_{3}(D)$ and therefore is 1 by $(2.5)$.

This completes the proof of the Theorem 1.1, apart from the claim that 5 is the best possible bound.

4. An example. Consider the group on three generators $a, b, c$ and with defining relators

$$
\begin{gathered}
a^{3}, b^{9}, c^{9},(b a)^{3},(c a)^{3},(a[c, b])^{3},[b, a, b] \\
{[b, c, b],[c, a, c],[c, b, c],[b c, a, b c],[b, a, c, c],[c, a, b, b] .}
\end{gathered}
$$

Using the computer program CAYLEY (Cannon [4]) we find the maximal nilpotent 3-quotient of exponent 9 of this group, to be of class 5 . Its order is $3^{13}$. A power-commutator presentation of this group, $H$ say, on thirteen generators can therefore be found, say $g_{1}, g_{2}, \ldots, g_{13}$ where $g_{1}$ is the image of $a$.

CAYLEY checks that $\left\langle g_{2}, g_{3}, \ldots, g_{13}\right\rangle$ is the Hughes subgroup $H_{3}(H)$ which is therefore proper.

Hence the proof of Theorem 2.11 is complete. Using (2.10) this also completes the proof of (1.1).

\section{REFERENCES}

1. R. Baer, Der Kern eine charakteristische Untergruppe. Compositio Math. 1 (1935), 254-283.

2. R. Baer, Gruppen mit Hamiltonschen Kern. Compositio Math. 2 (1935), 241-246.

3. R. Bryce, John Cossey and E. A. Ormerod, A note on p-groups with power automorphisms. Glasgow Math. J. 34 (1992), 327-332.

4. J. J. Cannon, An introduction to the Group Theory Language Cayley. Computational Group Theory (Durham 1982) pp. 145-183, (Academic Press, London-New York, 1984).

5. R. Dedekind, Über Gruppen, deren sämmtliche Theiler Normaltheiler sind. Math. Ann. 48 (1897), 548-561.

6. M. Hall, The Theory of Groups. (Macmillan, New York, 1959).

7. D. Hughes, Research Problem No. 3, Bull. Amer. Math. Soc. 63 (1957), 209.

8. F. W. Levi, Groups in which the commutator operation satisfies certain algebraic conditions. J. Indian Math. Soc. 6 (1942), 87-97. $345-360$.

9. Thomas Meixner, Power automorphisms of finite p-groups, Israel J. Math. 38 (1981), 
10. E. Schenkman, On the norm of a group. Illinois J. Math. 4 (1960), 150-152.

11. E. G. Strauss and G. Szekeres, On a problem of D. R. Hughes. Proc. Amer. Math. Soc. 9 (1958), 157-158.

Department of Mathematics

FACULTY OF SCIENCE

Australian National University

Canberra ACT 0200
Department of Mathematics

UNIVERSITY OF WESTERN SYDNEY, NEPEAN

P.O. Box 10

KingswoOd NSW 2747 